\title{
Dynamical Critical Exponents in Driven-Dissipative Quantum Systems
}

\author{
P. Comaron, ${ }^{1}$ G. Dagvadorj, ${ }^{2,3}$ A. Zamora, ${ }^{2}$ I. Carusotto, ${ }^{4}$ N. P. Proukakis, ${ }^{1}$ and M. H. Szymańska, ${ }^{2, *}$ \\ ${ }^{1}$ Joint Quantum Centre (JQC) Durham-Newcastle, School of Mathematics, Statistics and Physics, Newcastle University, \\ Newcastle upon Tyne, NE1 7RU, United Kingdom \\ ${ }^{2}$ Department of Physics and Astronomy, University College London, Gower Street, London, WC1E 6BT, United Kingdom \\ ${ }^{3}$ Department of Physics, University of Warwick, Coventry, CV4 7AL, United Kingdom \\ ${ }^{4}$ INO-CNR BEC Center and Dipartimento di Fisica, Università di Trento, 38123 Povo, Italy
}

(Received 22 August 2017; published 28 August 2018)

\begin{abstract}
We study the phase ordering of parametrically and incoherently driven microcavity polaritons after an infinitely rapid quench across the critical region. We confirm that the system, despite its driven-dissipative nature, satisfies the dynamical scaling hypothesis for both driving schemes by exhibiting self-similar patterns for the two-point correlator at late times of the phase ordering. We show that polaritons are characterized by the dynamical critical exponent $z \approx 2$ with topological defects playing a fundamental role in the dynamics, giving logarithmic corrections both to the power-law decay of the number of vortices and to the associated growth of the characteristic length scale.
\end{abstract}

DOI: 10.1103/PhysRevLett.121.095302

Two-dimensional (2D) driven-dissipative Bose systems display rich critical phenomena due to the interplay between drive, dissipation, and their collective phaseordering dynamics induced by the Bose degeneracy [1]. The picture is quite complex even at the level of the steady state, where the transition towards the ordered state has been predicted to be of either Kardar-Parisi-Zhang (KPZ) [2] or Berezinskii-Kosterlitz-Thouless (BKT) [3] type, depending on the subtle interplay between the drive and dissipation, and a finite size and effective anisotropy $[4,5]$. To date, the more advanced question of how continuous drive and dissipation affect the dynamics of nonequilibrium phase transitions remains largely open.

In this vein, one of the most useful concepts is the scaling hypothesis when the long-distance statistical properties of a complex system remain constant in time modulo a global change of scale [6]. While steady-state scaling behaviors are a generic feature of critical points, a dynamical scaling is found when the late-time dynamics after a quench is governed by a single characteristic length scale. Its value typically grows in time as a power law of elapsed time, the exponent being a universal quantity that does not feel the microscopic details but depends only on general features such as dimensionality, symmetry, and, of course, the equilibrium vs nonequilibrium condition.

Published by the American Physical Society under the terms of the Creative Commons Attribution 4.0 International license. Further distribution of this work must maintain attribution to the author(s) and the published article's title, journal citation, and DOI.
In the past decade or so, quantum gases of exciton polaritons in semiconductor microcavities have been widely used to study different aspects of the nonequilibrium Bose-Einstein condensation transition [7,8] and appear as a promising workhorse to investigate timedependent features for which theoretical predictions are still subject to debate. On general grounds, 2D drivendissipative systems described by KPZ-like phase dynamics are expected to show the dynamical critical exponent $z \approx$ 1.61 [9-11], while we expect $z=2$ when the KPZ nonlinearity ceases to be important. For the specific nonequilibrium case of microcavity polaritons, it has been suggested that the dynamical critical exponent $z$ takes values of either 1 or 2, depending on the system parameters [12], putting in question the universality of the phase ordering in this system.

In this Letter, we contribute to this exciting debate by exploring the dynamical critical properties of the phaseordering dynamics of polariton gases after an infinitely rapid quench from the disordered to deep in the (quasi) ordered $[6,13]$ phase. In particular, we look for scaling behaviors and associated universal exponents in the temporal evolution of the coherence length and of the density of topological defects (vortices). In order to capture the universal properties of the phase-ordering process, we study the polariton system in three among the most popular pumping regimes, namely, coherent pumping in the optical parametric oscillator (OPO) regime and the incoherent pumping (IP) with and without a frequency-selective pumping mechanism [7].

In spite of their marked differences, our calculations show strong numerical evidence that the critical behavior is the same in the three models, namely, a dynamical critical exponent $z \approx 2$ characteristic of a diffusive dynamics, plus 
logarithmic corrections. This is a good indication that refinement of the microscopic details of the model will not change the universal features properties: For the realistic system sizes considered in this work, the universal properties of the system, also in the dynamical case, are dominated by BKT type of physics in analogy to the static case considered recently theoretically [14] and experimentally [15], while the KPZ nonlinear terms do not play a role during dynamical crossing of the critical point.

System and method.-Our system consists of an ensemble of bosonic particles [excitons $(X)$ and photons $(C)$ for the parametrically pumped and lower polaritons (LP) for the incoherently driven case] with a finite lifetime interacting via contact interactions in two dimensions and driven in two distinct ways: parametrically and incoherently. The dynamical equations can be derived using the Keldysh field theory by including the classical fluctuations to all orders, but quantum fluctuations to the second order, which is appropriate in the long-wavelength limit, and employing the Martin-Siggia-Rose formalism (for a review, see [1]). An alternative derivation can be performed using FokkerPlanck equations for the Wigner function truncated to the third order $[7,16]$. Both methods lead to the same stochastic equation for the field $\psi(\mathbf{r}, t)$ with the noise term accounting for quantum and thermal fluctuations.

For the parametrically driven case, the finite grid version with $\hbar=1$ reads [14]

$i d\left(\begin{array}{c}\psi_{X} \\ \psi_{C}\end{array}\right)=d t\left[H_{\mathrm{MF}}\left(\begin{array}{c}\psi_{X} \\ \psi_{C}\end{array}\right)+\left(\begin{array}{c}0 \\ F_{p}\end{array}\right)\right]+\left(\begin{array}{c}\sqrt{\kappa_{X}} d W_{X} \\ \sqrt{\kappa_{C}} d W_{C}\end{array}\right)$,

where $\psi_{X, C}=\psi_{X, C}(\mathbf{r}, t)$ [with $\mathbf{r}=(x, y)$ ] are the exciton and cavity-photon fields, respectively, and $d W_{X}$ and $d W_{C}$ are the complex-valued zero-mean white Wiener noise terms with $\left\langle d W_{l}^{*}(\mathbf{r}, t) d W_{m}\left(\mathbf{r}^{\prime}, t\right)\right\rangle=\delta_{\mathbf{r}, \mathbf{r}^{\prime}} \delta_{l, m} d t$. The external monochromatic coherent pump $F_{p}=f_{p} e^{i\left(\mathbf{k}_{p} \cdot \mathbf{r}-\omega_{p} t\right)}$ injects photons with momentum $\mathbf{k}_{p}$ and frequency $\omega_{p}$ while both fields decay with their corresponding rates $\kappa_{X}$ and $\kappa_{C}$ : Here we use a shorthand notation

$$
H_{\mathrm{MF}}=\left(\begin{array}{cc}
\frac{-\nabla^{2}}{2 m_{X}}+g_{X}\left(\left|\psi_{X}\right|^{2}-\frac{1}{d V}\right)-i \kappa_{X} & \frac{\Omega_{R}}{2} \\
\frac{\Omega_{R}}{2} & \frac{-\nabla^{2}}{2 m_{C}}-i \kappa_{C}
\end{array}\right),
$$

with $m_{X}$ and $m_{C}\left(=2.3 \times 10^{-5} m_{e}\right)$ the exciton and cavityphoton masses, respectively. Since $m_{X} \gg m_{C}$, we consider the limit $m_{X} \rightarrow \infty$, and, consequently, the exciton field kinetic energy term disappears from Eq. (1). The excitonphoton Rabi splitting is given by $\Omega_{R}$ and the exciton-exciton interaction strength by $g_{X}$, and $d V=a^{2}$ is the numerical grid unit cell area (lattice spacing $a$ ). We solve Eq. (1) for parameters typical of current OPO experiments [7,14,17], namely, $\Omega_{R} \approx 4.4 \mathrm{meV}$ and $g_{X} \approx 2 \times 10^{-3} \mathrm{meV}_{\mu} \mathrm{m}^{2}$. We consider $\kappa_{X}=\kappa_{C}$, with $\kappa_{C}=1 / \tau_{C}$ and photon lifetime $\tau_{C}=6.58 \mathrm{ps}$. We set $\mathbf{k}_{p}=(1.6,0) \mu \mathrm{m}^{-1}$ and $\omega_{p}=\omega_{\mathrm{LP}}\left(\mathbf{k}_{p}\right)$, where $\omega_{\mathrm{LP}}$ is the lower-polariton dispersion. Here, we present results for a grid of $512^{2}$ lattice points with lattice spacing $a=0.87 \mu \mathrm{m}$ (total unit cell of $L_{x}=L_{y}=444.42 \mu \mathrm{m}$ ).

Since we are interested in the universal properties of driven-dissipative systems, we also consider the alternative typical setup of incoherent driving. Under the assumption that the high-energy reservoir follows adiabatically the condensate evolution and that the exciton and photon are locked into a single lower-polariton branch, the equation reads $(\hbar=1)[18]$

$$
\begin{aligned}
i d \psi_{\mathrm{LP}}= & d t\left[-\frac{\nabla^{2}}{2 m_{\mathrm{LP}}}+g_{\mathrm{LP}}\left|\psi_{\mathrm{LP}}\right|_{-}^{2}+\frac{i}{2}\left(\frac{P}{1+\frac{\left|\psi_{\mathrm{LP}}\right|_{-}^{2}}{n_{s}}}-\gamma_{\mathrm{LP}}\right)\right. \\
& \left.+\frac{1}{2} \frac{P}{\Omega} \frac{\partial}{\partial t}\right] \psi_{\mathrm{LP}}+\sqrt{\frac{P+\gamma_{\mathrm{LP}}}{4}} d W_{\mathrm{LP}}
\end{aligned}
$$

with $\psi_{\mathrm{LP}}=\psi_{\mathrm{LP}}(\mathbf{r}, t)$, and the lower-polariton field density reads (after subtracting the Wigner commutator contribution) $\left|\psi_{\mathrm{LP}}\right|_{-}^{2} \equiv\left(\left|\psi_{\mathrm{LP}}\right|^{2}-1 / 2 d V\right)$. The Wiener noise $d W_{\mathrm{LP}}$ has zero mean and fulfills $\left\langle d W_{\mathrm{LP}}^{*}(\mathbf{r}, t) d W_{\mathrm{LP}}\left(\mathbf{r}^{\prime}, t\right)\right\rangle=2 \delta_{\mathbf{r}, \mathbf{r}^{\prime}} d t$. $P$ defines the incoherent saturable and homogeneous driving strength and $n_{s}$ the saturation density, and we restrict ourselves to values $\left|\psi_{\mathrm{LP}}\right|_{-}^{2} \ll n_{s}$. We use typical experimental parameters [19]: $\gamma_{\mathrm{LP}}=1 / \tau_{\mathrm{LP}}$ with the polariton lifetime $\tau_{\mathrm{LP}}=4.5 \mathrm{ps}$, polariton mass $m_{\mathrm{LP}}=6.2 \times 10^{-5} m_{e}$, and polariton-polariton interaction strength $g_{\mathrm{LP}}=6.82 \times$ $10^{-3} \mathrm{meV} \mu \mathrm{m}^{2}$. To improve the physical relevance of the model when approaching the critical region, following Refs. [18,20], we implement frequency-selective pumping mechanism so that relaxation to low-energy modes is favored over energies higher than the cutoff frequency $\omega_{\text {cut }} \simeq$ $\Omega /\left[1+\left(\left|\psi_{\mathrm{LP}}\right|_{-}^{2}\right) / n_{s}\right]$ which are now suppressed. We use here $P / P_{\text {th }}=1.06$, where $P_{\text {th }}=\gamma_{\mathrm{LP}}$ is the mean-field critical pump, $\Omega=50 \gamma_{\mathrm{LP}}=11.09 \mathrm{ps}^{-1}$ and $n_{s}=$ $500 \mu \mathrm{m}^{-2}$ (labeled as $\mathrm{IP}_{\Omega=50}$ ) for the frequency-dependent pumping, and $P / P_{\text {th }}=1.1, \Omega=\infty$, and $n_{s}=1500 \mu \mathrm{m}^{-2}$ for the frequency-independent driving (labeled $\mathrm{IP}_{\Omega=\infty}$ ). We solve Eq. (2) in a square lattice of $301^{2}$ points, lengths $L_{x}=L_{y}=295.11 \mu \mathrm{m}$, and a periodic boundary condition and average over a sufficiently large number (400) of realizations in all schemes. The healing lengths $\xi=$ $1 / \sqrt{2 m_{C, \mathrm{LP}} g_{C, \mathrm{LP}}\left|\psi_{C, \mathrm{LP}}\right|_{-}^{2}}$ at the end of the evolution are, respectively, $\xi_{\Omega=50}^{\mathrm{IP}} \simeq 2.2 \mu \mathrm{m}, \quad \xi_{\Omega=\infty}^{\mathrm{IP}} \simeq 0.8 \mu \mathrm{m}$, and $\xi^{\mathrm{OPO}} \simeq 1.84 \mu \mathrm{m}$, with the condition $L(t) \gg \xi$ fulfilled in all cases [21].

In both models, a nonequilibrium steady state with finite particle density $\left|\psi_{X, C, \mathrm{LP}}\right|^{2}$ is established once the pumping strength overcomes the cavity losses. Also, by tuning the 
strength of the pump power, both the OPO and the IP system (with or without a frequency-dependent pumping mechanism) undergo a BKT type of phase transition between a disordered phase with an exponential and an ordered phase with a power-law decay of spatial coherence, governed by the binding or unbinding of vortex-antivortex pairs (see Fig. 1). For the parametric pumping, the BKT transition in the steady state is analyzed in detail in Ref. [14]. A similar transition takes place for the IP system (Fig. 1, bottom). It is worth noting that, in this case, stronger fluctuations at higher modes $(\Omega \rightarrow \infty)$ and a smaller saturation density $\left(n_{s} \rightarrow 0\right)$ lead to a larger shift of the pump power of the BKT transition with respect to the mean-field onset of macroscopic population growth.
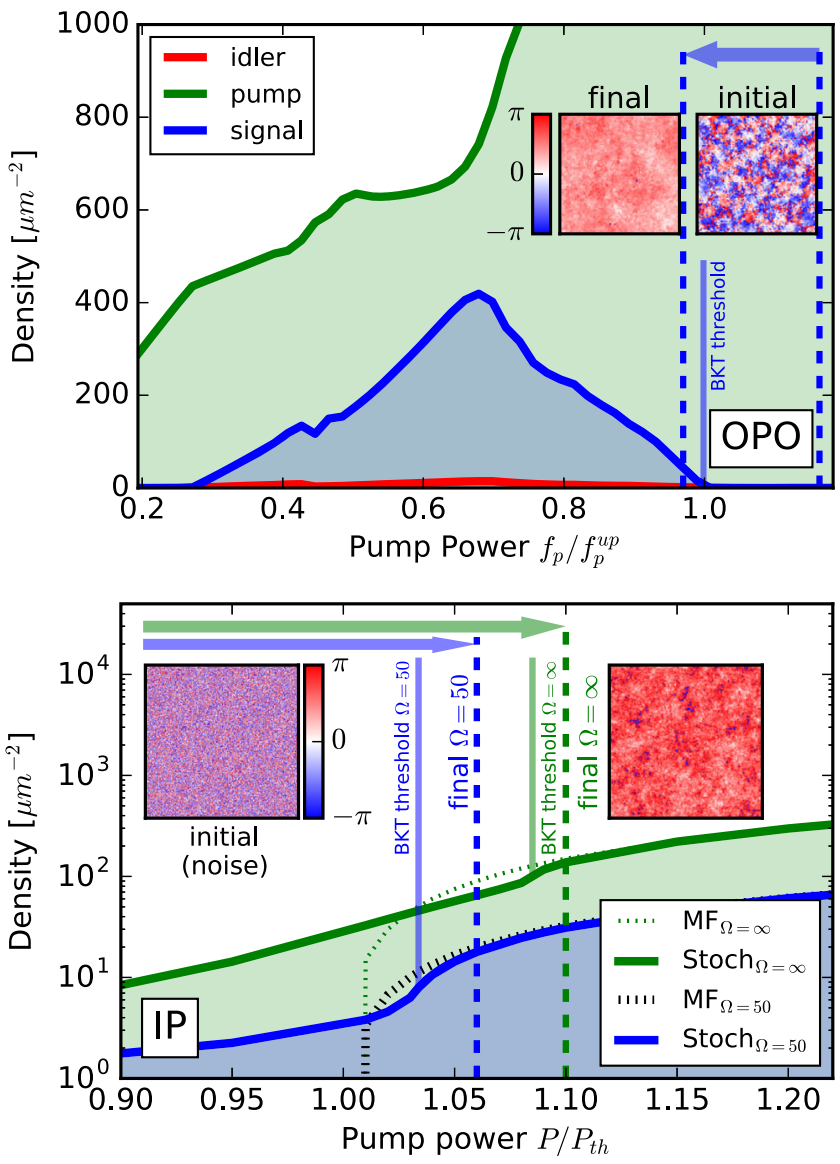

FIG. 1. BKT transition with parametric and incoherent pumping. Top panel: Noise-averaged density from stochastic equations of the signal (blue), idler (red), and pump (green) as a function of pump power $f_{p}$ for parametrically driven polaritons across OPO. Bottom panel: Mean-field (dotted lines) and noise-averaged (solid lines) densities for frequency-independent (green) and frequency-dependent (blue) incoherent pumping. In both panels, arrows indicate the infinitely rapid quench protocol across the critical region, and pump powers are scaled to their corresponding mean-field threshold values. The insets show typical snapshots of the real space phase profile for the initial and late-time states.
Universal dynamical scaling.-We now study an infinitely rapid quench across a critical point. For the parametrically pumped case (see Fig. 1, top), we quench through the upper critical threshold $f_{p}^{\text {up }}$. The system is prepared in the steady state of a deeply disordered phase at a given pump power $f_{p}^{i}>f_{p}^{\text {up }}$, where bound and unbound vortices proliferate (see the inset), and is instantaneously quenched to a deep quasiordered regime by adjusting the external drive to $f_{p}^{f}$, with $f_{p}^{f}<f_{p}^{\text {up }}$. For the incoherently pumped case (see Fig. 1, bottom), we quench from the random initial configuration deep in the disordered phase (i.e., steady state for $P^{i}=0$ ) to a quasiordered regime by setting the external drive to $P^{f}>P_{\mathrm{th}}$ at $t=0$ and letting the system evolve. We explore the dynamical scaling properties of the system during the phase ordering by considering the first-order two-point correlation function [14]:

$$
g^{(1)}(\mathbf{r}, t)=\frac{\left\langle\psi^{*}(\mathbf{r}+\mathbf{u}, t) \psi(\mathbf{u}, t)\right\rangle-\delta_{\mathbf{r}+\mathbf{u}, \mathbf{u}} / 2 d V}{\sqrt{\left\langle|\psi(\mathbf{r}+\mathbf{u}, t)|_{-}^{2}\right\rangle\left\langle|\psi(\mathbf{u}, t)|_{-}^{2}\right\rangle}},
$$

where $\langle\ldots\rangle$ denotes averaging over both noise realizations and the auxiliary position $\mathbf{u}$ and $t$ is the time after the quench. For the parametrically pumped scheme, $\psi$ in Eq. (3) corresponds to the signal, which is obtained by filtering the cavityphoton field $\psi_{C}$ from Eq. (1) around the signal momentum $\mathbf{k}_{s}$. For the incoherently pumped system, $\psi$ is the polariton field $\psi_{\mathrm{LP}}$.

In the phase-ordering kinetics of the planar $X Y$ model in two dimensions $[24,25,28]$, the non-steady-state twopoint correlator (3) fulfills the dynamical scaling form $g^{(1)}(r, t) \sim g_{\mathrm{SS}}^{(1)}(r, t) F[r / L(t)]$, with the steady-state correlation function decaying algebraically at long distances, as $g_{\mathrm{SS}}^{(1)}(r) \sim r^{-\alpha}$. The scaling function $F$ tends to 1 when $r \ll L(t)$, indicating that the critical correlations have been established at distances much smaller than $L(t)$ at time $t$, which defines the characteristic length scale of the system $L(t)$. Since our system is highly nonequilibrium, it is far from obvious whether similar scaling behavior holds here in the presence of strong drive and dissipation. Indeed, we obtain a perfect collapse when plotting the two-point correlation function $g^{(1)} / g_{\mathrm{SS}}^{(1)}$ as a function of the rescaled length $r / L(t)$ at different times of the late dynamics for both driving schemes (see Fig. 2). For consistency, we extract the length scale $L(t)$ when $\left(g^{(1)} / g_{\mathrm{SS}}^{(1)}\right)(L(t), t)=$ 0.5 (white dots in insets in Fig. 2), with the independence of our conclusions on the intersection value verified in Supplemental Material [21].

This collapse of the two-point correlation function is a strong indication that our polariton system fulfills the scaling hypothesis. We can then access the universal dynamical critical exponent $z$ of our driven-dissipative system by analyzing the growth of the characteristic length $L(t)$ and the decay of the number of topological defects (vortices) at late times after a sudden quench. Note that an 


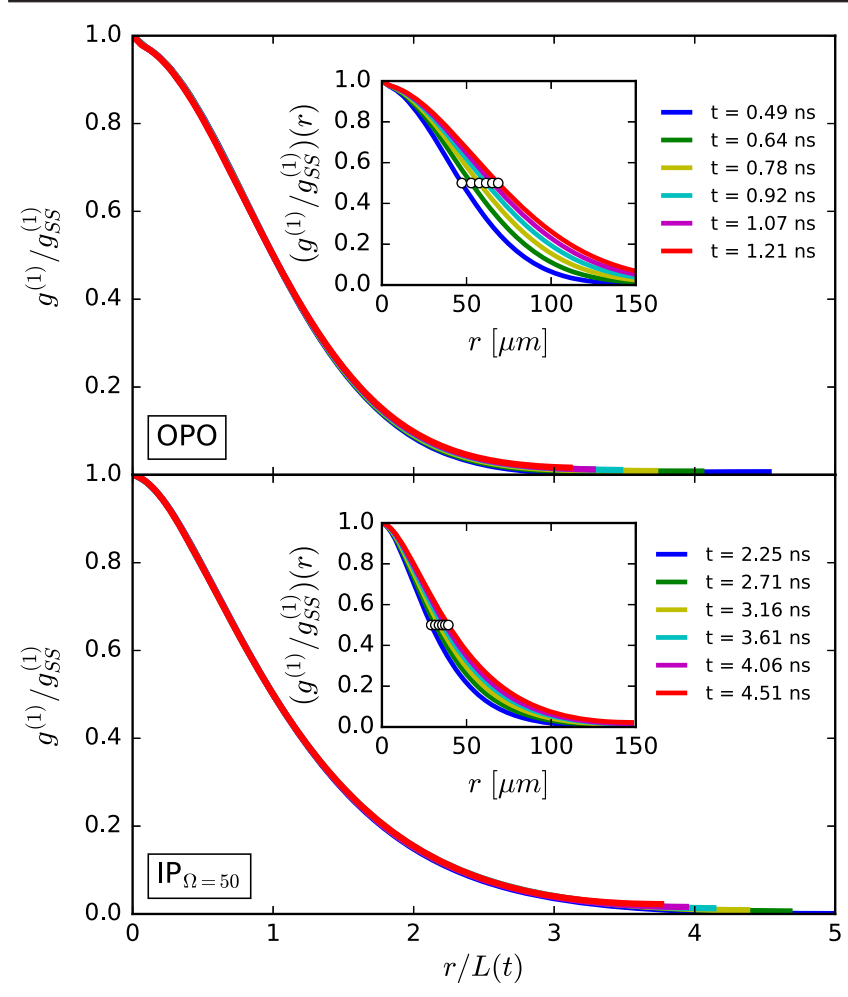

FIG. 2. Scaling of the two-point correlation function. Firstorder correlation function $g^{(1)}$ (normalized by the corresponding steady state correlator $g_{\mathrm{SS}}^{(1)}$ ) for the parametrically pumped (top) and incoherently pumped polaritons with (bottom) frequencydependent pumping as a function of the rescaled distance $r / L(t)$ at different times during the phase-ordering process. The evident collapse of the curves confirms the dynamic scaling hypothesis. The insets show $g^{(1)} / g_{\text {SS }}^{(1)}$ at different times, from which the characteristic length scale $L(t)$ is obtained by considering $\left(g^{(1)} / g_{\mathrm{SS}}^{(1)}\right)(L(t), t)=0.5$ (white dots).

equilibrium analogue of the phase degree of freedom for the polariton system is the planar $X Y$ model, where free vortices and bound vortex-antivortex pairs exist even in the steady state below and above the BKT phase transition, respectively. The existence of the steady-state vortices plays a fundamental role in the phase-ordering process and introduces the characteristic logarithmic correction into the late-time dynamics of both $L(t)$ and a number of vortices $n_{v}$ following a sudden quench [24,29], such that $L(t) \sim\left(\left(t / t_{0}\right) / \log \left(t / t_{0}\right)\right)^{1 / z}$ and $n_{v}(t) \sim\left(\left(t / t_{0}\right) /\right.$ $\left.\log \left(t / t_{0}\right)\right)^{-(2 / z)}$, where $t_{0}$ is a nonuniversal microscopic system timescale (taken here as $t_{0}=1 \mathrm{ps}$ [21]). The two relations follow from the fact that $n_{v}(t) \sim 1 / L(t)^{2}$ when there is a unique length scale in the system, which is true at late times in the dynamics [21].

To demonstrate the emergence of this universal scaling from our numerical data, Fig. 3 plots the characteristic length $L(t)$ and number of vortices $n_{v}(t)$ for the three different pumping schemes considered. The OPO (top) and $\mathrm{IP}_{\Omega=\infty}$ (center) cases are qualitatively identical in the sense
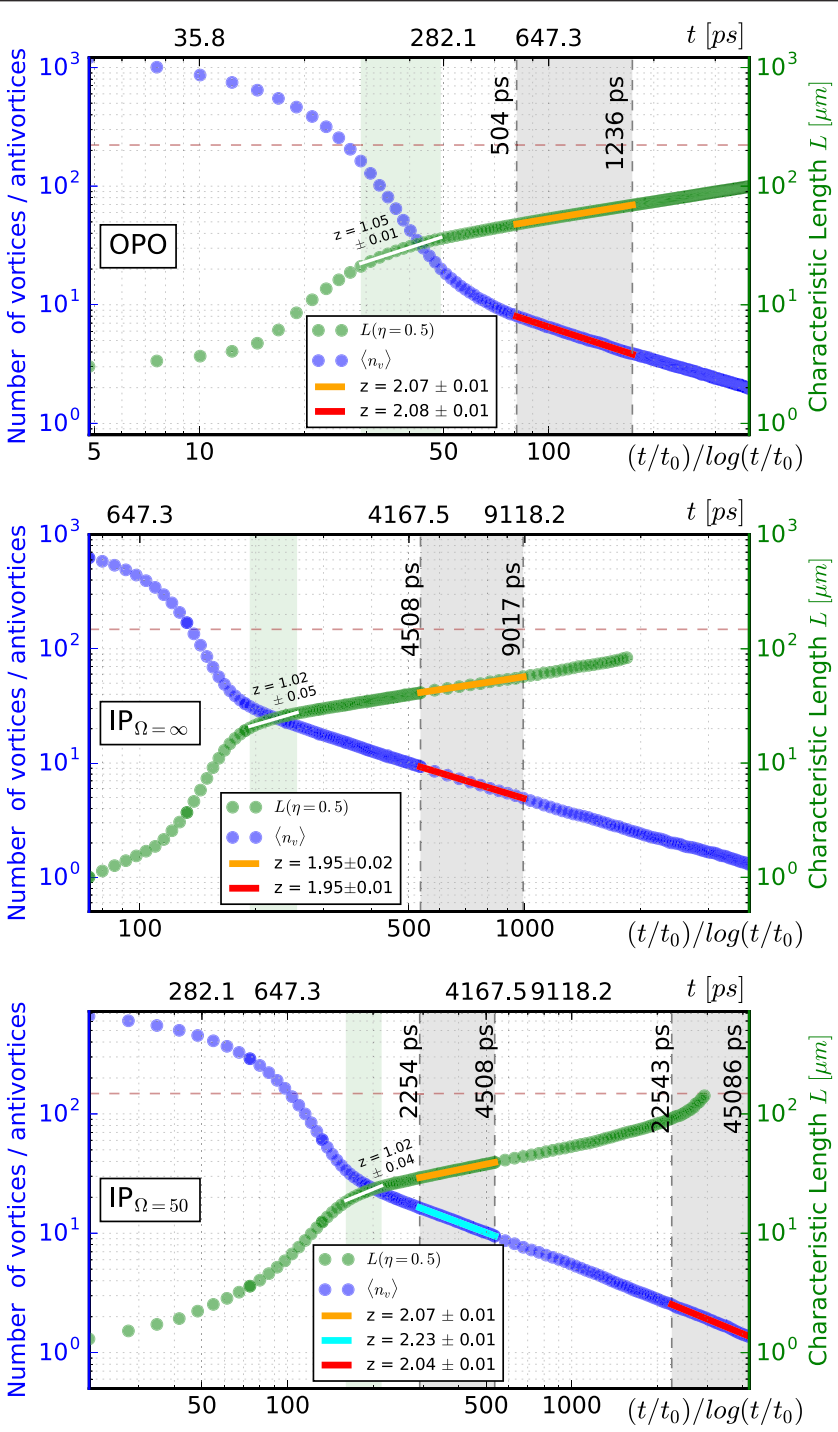

FIG. 3. Topological defects and $L(t)$ during phase ordering. Density of vortices (blue) and the characteristic length scale $L(t)$ (green) as a function of time after an infinite rapid quench for parametric (top panel), frequency-independent (central panel), and frequency-dependent (bottom panel) incoherently pumped polaritons. The size of the numerical grid is marked by the horizontal (brown) dashed lines. The late-time dynamics show characteristic diffusive behavior described by logarithmic corrections $\left(t / t_{0}\right) / \log \left(t / t_{0}\right)$ to the dominant power-law scaling due to the presence of the topological defects. For all configurations, we obtain a nonequilibrium dynamical critical exponent $z \approx 2$ within the gray-shaded regions.

that $g^{(1)}$ collapses in the same time window as that in which the respective vortex dynamics reaches the converged $z \approx 2$ value. We are careful to fit $L(t)$ and $n_{v}$ late enough in the quench so the dynamics becomes universal (indeed, both reveal $z \approx 2$ ) but before size effects, power-law correlations, or a very small vortex number affect our analysis. However, in the case of $\operatorname{IP}_{\Omega=50}$ (bottom), strong damping of collective fluctuations, introduced by the explicitly frequency-dependent nature of the pump, is responsible for 
the collective modes to reach $z \approx 2$ at a much earlier time than the topological modes. Nevertheless, both channels show the dynamical critical exponents to be $z \approx 2$ in their appropriate late-time windows. A more detailed discussion about the fitting criteria can be found in Supplemental Material [21].

We stress that the sufficiently late-time analysis is essential to allow all channels to equilibrate properly and fulfill the scaling hypothesis, whereas fitting early in the phase-ordering process, and before the dynamics becomes universal (light green regions in Fig. 3), can lead to the incorrect conclusion of $z \approx 1$ [30]. Note that, for the experimentally realistic parameters considered in our simulations, the phase ordering takes place on timescales similar to the polariton population growth [21], which is the case for which Ref. [12] predicted $z \approx 1$.

Summary and outlook.-In this work, we have theoretically studied the universal critical properties of twodimensional driven-dissipative Bose systems after an infinitely rapid quench into a quasiordered phase. Focusing on exciton-polariton gases in semiconductor microcavities under different pumping configurations, our work reveals clearly that universal physics emerges in the late-time, long-distance dynamics of both the topological defects and smooth phase fluctuations, whereas the nonuniversal early dynamics can give misleading information about the critical exponents. The required system size in the $100 \mu \mathrm{m}$ range needed to access this physics appears reachable in the new generation of samples [15,31]. In particular, our $z \approx 2$ prediction for the dynamical critical exponent of realistic size systems is different from the ones of conservative Bose systems [26,27,32,33] and of nonequilibrium KPZ systems and rather recovers a diffusive dynamics in the planar $X Y$ model $[24,25,28]$.

Additional metadata are available by following the link in Ref. [34].

We thank George W. Stagg and Kean Loon Lee for computational assistance and Michal Matuszewski and Leticia Cugliandolo for fruitful discussions. We acknowledge financial support from Engineering and Physical Science Research Council: Grants No. EP/I028900/2 and No. EP/ K003623/2 (A. Z., G. D., and M.H. S.) and EP/L504828/1 (P. C. and N. P. P. for DTA support). This work was supported by the EU-FET Proactive grant AQuS, Project No. 640800, and by the Autonomous Province of Trento, partially through the project "On silicon chip quantum optics for quantum computing and secure communications (SiQuro)". Data supporting this publication are openly available under an "Open Data Commons Open Database License".

*m.szymanska@ucl.ac.uk

[1] L. M. Sieberer, M. Buchhold, and S. Diehl, Rep. Prog. Phys. 79, 096001 (2016).
[2] M. Kardar, G. Parisi, and Y. C. Zhang, Phys. Rev. Lett. 56, 889 (1986).

[3] J. M. Kosterlitz and D. J. Thouless, J. Phys. C 6, 1181 (1973).

[4] E. Altman, L. M. Sieberer, L. Chen, S. Diehl, and J. Toner, Phys. Rev. X 5, 011017 (2015).

[5] A. Zamora, L. M. Sieberer, K. Dunnett, S. Diehl, and M. H. Szymanska, Phys. Rev. X 7, 041006 (2017).

[6] A. J. Bray, Adv. Phys. 51, 481 (2002).

[7] I. Carusotto and C. Ciuti, Rev. Mod. Phys. 85, 299 (2013).

[8] J. Keeling, F. M. Marchetti, M. H. Szymańska, and P. B. Littlewood, Semicond. Sci. Technol. 22, R1 (2007).

[9] A. Pagnani and G. Parisi, Phys. Rev. E 92, 010101 (2015).

[10] L. Canet, H. Chaté, B. Delamotte, and N. Wschebor, Phys. Rev. Lett. 104, 150601 (2010).

[11] T. Halpin-Healy and G. Palasantzas, Europhys. Lett. 105, 50001 (2014).

[12] M. Kulczykowski and M. Matuszewski, Phys. Rev. B 95, 075306 (2017).

[13] P. C. Hohenberg and B. I. Halperin, Rev. Mod. Phys. 49, 435 (1977).

[14] G. Dagvadorj, J. M. Fellows, S. Matyjaśkiewicz, F. M. Marchetti, I. Carusotto, and M. H. Szymańska, Phys. Rev. X 5, 041028 (2015).

[15] D. Caputo, D. Ballarini, G. Dagvadorj, C. Sánchez Muñoz, M. De Giorgi, L. Dominici, K. West, L. N. Pfeiffer, G. Gigli, M. H. Szymańska, and D. Sanvitto, Nat. Mater. 17, 145 (2018).

[16] I. Carusotto and C. Ciuti, Phys. Rev. B 72, 125335 (2005).

[17] D. Sanvitto et al., Nat. Phys. 6, 527 (2010).

[18] A. Chiocchetta and I. Carusotto, Europhys. Lett. 102, 67007 (2013).

[19] W. H. Nitsche, N. Y. Kim, G. Roumpos, C. Schneider, M. Kamp, S. Höfling, A. Forchel, and Y. Yamamoto, Phys. Rev. B 90, 205430 (2014).

[20] M. Wouters and I. Carusotto, Phys. Rev. Lett. 105, 020602 (2010).

[21] See Supplemental Material at http://link.aps.org/ supplemental/10.1103/PhysRevLett.121.095302 for a discussion on numerical methods and convergence, vortex number dynamics, logarithmic correction, self-consistency of characteristic length, and dependence of the dynamical exponent on the intersection point and microscopic system timescale. Supplemental Material includes Refs. [7,12,14,19,22-27].

[22] J. Kasprzak, M. Richard, S. Kundermann, A. Baas, P. Jeambrun, J. Keeling, F. M. Marchetti, M. H. Szymańska, R. André, J. L. Staehli, P. B. Savona, B. Littlewood, R. Deveaud, and Le Si Dang, Nature (London) 443, 409 (2006).

[23] G. R. Dennis, J. Hope, and M. T. Johnsson, Comput. Phys. Commun. 184, 201 (2013).

[24] A. Jelić and L. F. Cugliandolo, J. Stat. Mech. (2011) P02032.

[25] A. J. Bray, A. J. Briant, and D. K. Jervis, Phys. Rev. Lett. 84, 1503 (2000).

[26] L. A. Williamson and P. B. Blakie, Phys. Rev. Lett. 116, 025301 (2016).

[27] K. Damle, S. N. Majumdar, and S. Sachdev, Phys. Rev. A 54, 5037 (1996).

[28] A. D. Rutenberg and A. J. Bray, Phys. Rev. E 51, R1641 (1995).

[29] B. Yurke, A. N. Pargellis, T. Kovacs, and D. A. Huse, Phys. Rev. E 47, 1525 (1993). 
[30] Note that the universality of this value is already ruled out by the dependence of the $g^{(1)}$ collapse on the intersection value used to extract $L(t)$.

[31] F. Baboux et al., arXiv:1707.05798.

[32] K. Kudo and Y. Kawaguchi, Phys. Rev. A 88, 013630 (2013).
[33] J. Hofmann, S. S. Natu, and S. DasSarma, Phys. Rev. Lett. 113, 095702 (2014).

[34] See http://discovery.ucl.ac.uk/10051965. Please contact UCL Open Access http://www.ucl.ac.uk/library/openaccess/contact-us for access instructions. 